PALEO

Revue d'archéologie préhistorique

30-1 | 2019

Varia

\title{
Exploitation de la marmotte dans le niveau Magdalénien de La Chênelaz (Hostiaz, Ain, France)
}

Exploitation of the Marmot in the Magdalenian level of La Chênelaz (Hostiaz, Ain, France)

Ingrid Gay, Hervé Monchot, Cédric Beauval et Marc Cartonnet

\section{OpenEdition}

Journals

Édition électronique

URL : http://journals.openedition.org/paleo/4572

DOI : $10.4000 /$ paleo.4572

ISSN : 2101-0420

Éditeur

SAMRA

Édition imprimée

Date de publication : 30 décembre 2019

Pagination : 148-162

ISSN : 1145-3370

Référence électronique

Ingrid Gay, Hervé Monchot, Cédric Beauval et Marc Cartonnet, « Exploitation de la marmotte dans le niveau Magdalénien de La Chênelaz (Hostiaz, Ain, France) », PALEO [En ligne], 30-1 | 2019, mis en ligne le 29 mai 2020, consulté le 08 juillet 2020. URL : http://journals.openedition.org/paleo/4572 ; DOI : https://doi.org/10.4000/paleo.4572

\section{(c) (1) $\odot \Theta$}

PALEO est mis à disposition selon les termes de la licence Creative Commons Attribution - Pas d'Utilisation Commerciale - Pas de Modification 4.0 International. 
EXPLOITATION

DE LA MARMOTTE DANS

LE NIVEAU MAGDALÉNIEN

DE LA CHÊNELAZ

(HOSTIAZ, AIN, FRANCE)

Ingrid Gaya,

Hervé Monchotb,

Cédric Beauvalc

Marc Cartonnet ${ }^{\mathrm{d}}$

a. Aix Marseille Université, CNRS, MCC, LAMPEA UMR 7269, Maison

Méditerranéenne des Sciences de l'Homme, 5 rue du Château de l'horloge, BP 647, FR-13094, Aix-en-Provence.

b. UMR 8167 « Orient \& Méditerranée », 27 rue Paul Bert, FR-94200 Ivry-sur-

Seine-herve.monchot@wanadoo.fr

c. Archéosphère, 10 rue de la Rhode, FR-11500 Quillan

c.beauval@archeosphere.com

d. 198 Montée de Millieu, FR-01680 Lhuis

PALE0 30 | t. 1

DÉCEMBRE 2019

PAGES 148 À 162

MOTS-CLÉS Marmotte des Alpes (Marmota marmota), profil squelettique, profil de mortalité, saison d'abattage, Tardiglaciaire.
Bien qu'occasionnelle, l'exploitation de la marmotte à but alimentaire ou artisanal (travail des fourrures), a été bien documentée en Europe notamment dans les Alpes au cours des temps préhistoriques. C'est aussi le cas sur les hauts plateaux jurassiens qui se caractérisent par des conditions géomorphologiques et écologiques spécifiques permettant au Magdalénien la mise en place de systèmes de subsistance saisonniers. Cet article présente une étude archéozoologique (représentation squelettique, profil de mortalité, saisonnalité d'abattage, traces de découpe) des restes de marmottes de la grotte de la Chênelaz dans le sud du Jura (Hostiaz, Ain) montrant ainsi la place de cet animal dans l'économie locale au Magdalénien. 
Exploitation of the Marmot in the Magdalenian level of La Chênelaz (Hostiaz, Ain, France)

Although occasional, the exploitation of the marmot for food, but also for fur, has been well documented in Europe especially in the Alps during prehistoric times. This is also the case in the Jura highlands, which are characterized by specific morphological and ecological conditions that make it possible for the Magdalenian to set up a seasonal subsistence system. This article presents an archaeozoological study (skeletal representation, mortality profile, slaughter season, butchery marks) of the marmot remains from the Chênelaz cave in the Jura (Hostiaz, Ain) thus showing the important place of this animal in the local economy during the Magdalenian.

\section{INTRODUCTION}

La marmotte alpine (Marmota marmota Linné, 1758) fait partie des petits gibiers dont la présence dans une série archéologique interpelle. En effet, en Europe, ce mammifère n'occupe aujourd'hui qu'un seul type de biotope, des zones herbeuses situées au-dessus de la limite supérieure de la forêt entre 1300 et 3000 mètres d'altitude (Mann et al. 1993 ; Ramousse et al. 1999). Cette amplitude altitudinale pourrait être due à la fraîcheur hivernale qu'elle recherche, ainsi qu'à la pression exercée par l'homme sur l'espèce. Ainsi au Paléolithique, des restes de marmottes ont été identifiés dans les plaines du Bassin parisien (Chaline 1972), dans le Massif-Central (e.g. La Chapelle-aux-Saints, Beauval et al. 2004) et plus à l'ouest en Poitou-Charentes (Tournepiche 1996 ; Airvaux et al. 2012) jusqu'à Jonzac, à 40 m d'altitude (Beauval 2004). La marmotte est néanmoins un marqueur bioclimatique intéressant notamment pour le Tardiglaciaire (Bintz et Griggo 2011). Les premiers travaux sur la marmotte remontent au début du XXe siècle avec Hippolyte Müller, fondateur du Musée dauphinois à Grenoble, qui a notamment fouillé entre 1903 et 1921 quatre sites tardiglaciaires dans le Vercors (Müller 1914, 1917 ; Monin et al. 2007)1. Puis il faut attendre la fin des années 1980, pour voir la publication de différentes études qui ont permis de montrer l'importance de la marmotte dans l'économie de subsistance des chasseurs-cueilleurs du Tardiglaciaire dans l'arc alpin (e.g. Patou 1987 ; Desbrosse et al. 1991 ; Giacobini 1992 ; Pohar 1997 ; Tomé 1998, 2005 ; Cartonnet et Naton 2000 ; Fournier 2005 ; Griggo et al. 2012 ; Monin et al. 2006, 2010 ; Tomé et Chaix 2003 ; Peresani 2008 ; Gurioli et al. 2011; Romandini et al. 2012). Lorsque l'origine anthropique d'une série est avérée (i.e. chasse), elle met en évidence une acquisition spécifique ainsi qu'un traitement de la carcasse pour la récupération de produits tels que la viande, la graisse et la fourrure, tout comme celle observée pour les grands mammifères (renne, bouquetin, cheval ou encore bison).

Cet article présente une étude archéozoologique des restes de marmottes de la grotte de la Chênelaz dans le Bugey (sud du Jura, Hostiaz, Ain), montrant ainsi la place importante de ce petit mammifère dans l'économie locale au Magdalénien. Ce travail sur les marmottes de la Chênelaz a été mené dans le cadre d'une thèse de doctorat sur La saisonnalité des occupations humaines au Tardiglaciaire dans les Alpes occidentales, comprenant l'étude de plusieurs sites du tardiglaciaire alpin où la marmotte était l'un des taxons prédominant: Colomb (Méaudre), la Passagère (Méaudre), l'Olette (Lans-enVercors), les Freydières (Saint-Agnan-en-Vercors), Bobache (La Chapelle-en-Vercors), Jean-Pierre 1 (Saint-Thibaud-deCouz) (Gay 2015). Une grande partie de cette thèse a été dédiée à l'analyse de la saisonnalité et à l'élaboration de référentiels, non seulement sur la marmotte, mais aussi sur le bouquetin (Capra ibex). Les données sur la Chênelaz seront mises en regard avec les résultats obtenus sur les sites préalpins.

\section{KEY-WORDS Alpine marmot (Marmota marmota), skeletal profile, ageing, slaughter season, Late glacial.}

[1] Dès la fin du XIXe siècle, des restes de marmottes ont été décrits au sein de formations fossilifères pléistocènes (Breuil 1899 ; Laville 1908). 


\section{1 | PRÉSENTATION GÉNÉRALE DU SITE}

\section{1 | Localisation et topographie}

La grotte de la Chênelaz est située à 900 m d'altitude, sur le territoire communal d'Hostiaz. Elle se développe en limite d'un plateau qui surplombe la cluse des Hôpitaux et s'adosse au massif de la Charbonnière culminant à 1065 m (fig. 1). Les calcaires de l'oxfordien supérieur, dominant la cluse des Hôpitaux et les gorges de l'Albarine, façonnent une vaste étendue karstique au pied de la cavité. Le réseau fossile de la Chênelaz progresse quant à lui dans des calcaires construits à polypiers du kimméridgien supérieur. Un miroir de faille, orienté estouest semble être à l'origine de la formation de cette petite cavité (Naton 1995).

La grotte s'articule autour de deux salles séparées par un corridor bâti autour d'un verrou rocheux (fig. 2). Le porche d'entrée en forme d'arche, orienté sud-ouest, donne accès à la première salle de section sub-rectangulaire mesurant $8 \mathrm{~m}$ de longueur pour une largeur moyenne de $6 \mathrm{~m}$. Le remplissage, avec un maximum de $90 \mathrm{~cm}$ d'épaisseur, repose directement sur le substratum calcaire très érodé. La salle 2, en forme de puits circulaire, s'incline en direction du fond de la cavité et contient une accumulation de plusieurs mètres d'épaisseur de sédiments. La surface globale au sol couvre $33 \mathrm{~m}^{2}$. Une galerie basse (ancien siphon) dans la zone profonde de la salle, actuellement en majeure partie colmatée, contient des sédiments endokarstiques. Ces derniers ont été déposés lors d'une ancienne activité hydrologique. Cette grotte a donc aussi fonctionné comme une exsurgence.

\section{2 | Stratigraphie}

Deux coupes stratigraphiques schématiques, la première à l'aplomb du porche, la seconde dans le milieu de la salle 2, permettent d'en synthétiser le remplissage. Dans la salle 2 , un sondage de $3 \mathrm{~m}$ de profondeur n'a pas permis d'atteindre le plancher de la cavité (fig.3). À la base du

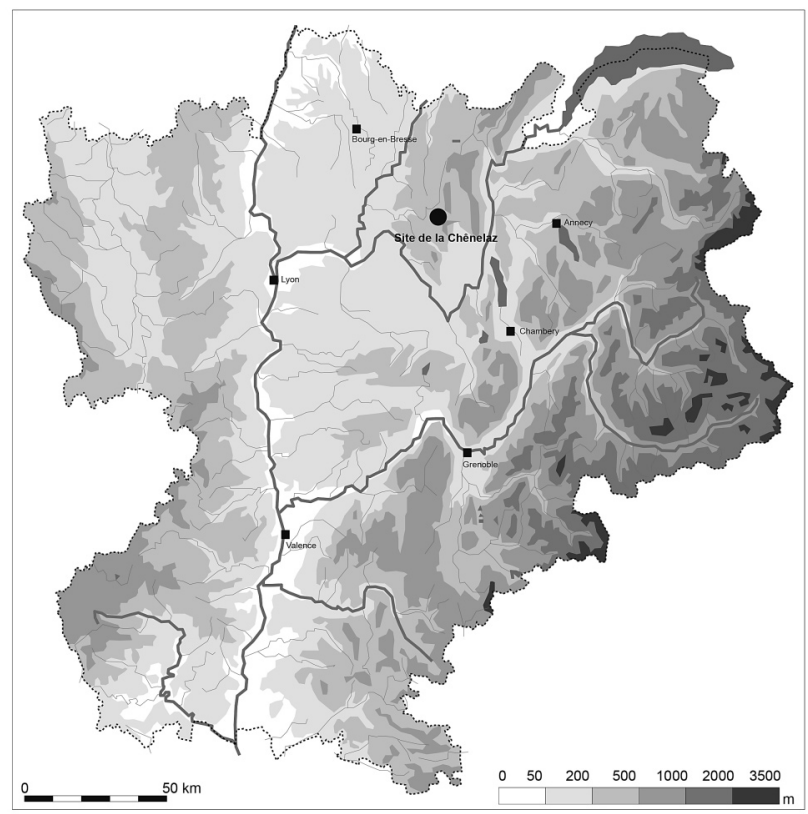

remplissage, les couches sont essentiellement composées par des sédiments très fins, d'origine endokarstique (couches 5 à 6). Au-dessus, elles sont constituées par des éléments provenant majoritairement de la desquamation des parois (couches 1 à 4). Seules les couches anthropiques feront l'objet d'une description.

Les unités stratigraphiques $5 a$, 5b et $6 a, 6 b, 6 c$ présentent plusieurs encroutements de calcite séparant nettement les différents niveaux. Dans ces unités stratigraphiques, des artefacts moustériens ont été trouvés associés à une faune diversifiée et abondante, largement dominées par l'ours des cavernes (Ursus spelaeus) et l'ours brun (Ursus arctos) (Schweizer 2002 ; Monchot et Beauval inédit).

Le dépôt du niveau 2c est formé par des sédiments sabloargileux emballant un cailloutis dense, il contient l'occupation magdalénienne. La couche $2 \mathrm{~b}$ présente moins de cailloux et une matrice argileuse plus abondante. Les phénomènes d'altération et de corrosion sont plus forts. Les mésolithiques se sont installés dans le site à cette période. La couche 2 a est un cailloutis mal colmaté. On y trouve des vestiges du début de l'Age du Fer. Enfin, le cailloutis cryoclastique constituant la couche 1 renferme des vestiges gallo-romains et médiévaux.

La seconde coupe, relevée à l'aplomb du porche d'entrée, mesure $90 \mathrm{~cm}$ de profondeur. Trois couches, surtout composées d'apports épikarstiques et exogènes, ont été distinguées. Seule la couche 3, constituée essentiellement d'argile jaune orangé très homogène pourrait correspondre au colmatage du fond de la salle 2. Ce sédiment repose directement sur le substratum de la salle d'entrée. La couche 2 est un cailloutis dense avec quelques gros blocs calcaires englobés dans une matrice de couleur marron sombre, elle contient l'occupation magdalénienne. La couche 1 composée d'un sédiment cendreux gris foncé emballe un cailloutis fortement corrodé. Cette couche de surface a subi de nombreuses perturbations et associe les vestiges protohistoriques et historiques (Cartonnet et Naton 2000).

\section{FIGURE 1}

Carte de situation géographique de la grotte de la Chênelaz (Hostiaz, Ain). Location map of the Chênelaz cave (Hostiaz, Ain). 


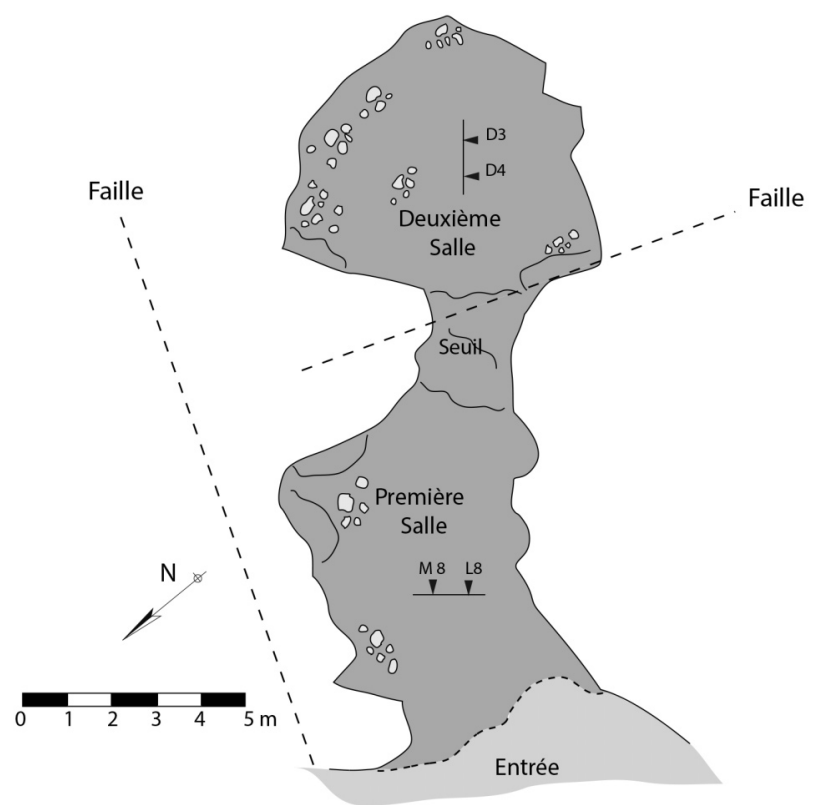

\section{FIGURE 2}

Plan et localisation des coupes stratigraphiques de la grotte de la Chênelaz (Hostiaz, Ain).

Plan and and location of the stratigraphic sections of Chênelaz cave (Hostiaz, Ain).

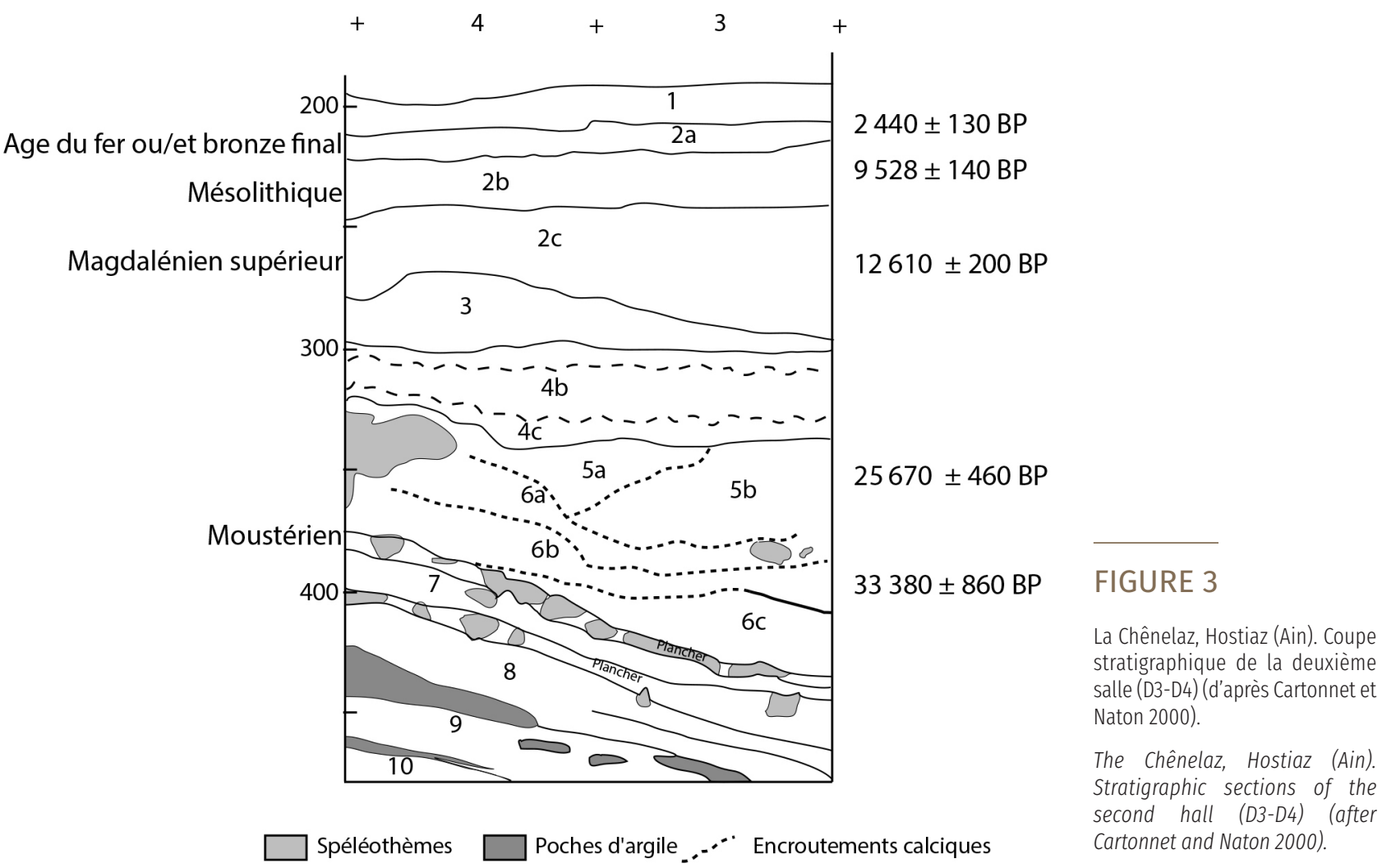

\section{3 | Agencement spatial de l'occupation magdalénienne}

\subsubsection{La salle d'entrée et le porche}

L'occupation magdalénienne couvre l'ensemble du site et l'espace semble avoir été délimité pour plusieurs activités. Deux dates ont été obtenues au niveau de la salle d'entrée (Cartonnet et Naton 2000) : une incisive de marmotte a été datée $12190 \pm 80$ B.P. (Ly 383/OxA 6427) et un fragment de renne $12460 \pm 65$ B.P. (Ly 703/OxA 8027). Dans la salle d'entrée et le porche, une petite unité domestique accompagne une importante zone de débitage, bien structurée. Sa surface de $7 \mathrm{~m}^{2}$ fait face à la lumière naturelle. La mise en forme préparatoire des nucléus semble avoir été amorcée en dehors du site, probablement dans les zones de collecte de matière première. La production d'éléments prédéterminés, lames et lamelles, domine avec pour objectif la fabrication d'armatures, essentiellement des lamelles à bord abattu. Par ailleurs, l'outillage de fond commun est façonné sur des lames et des éclats laminaires. Les restes de marmottes sont rares (NRD = 43 pour un NMI de 1). À ce jour, aucune structure de combustion n'a été constatée. 


\subsection{2 | La salle du fond}

La salle du fond a été fouillée dans sa globalité. Le niveau magdalénien (2c) est documenté par deux dates: la première sur os de marmotte (Ly 4790) $12610 \pm 200$ B.P et la seconde sur une incisive de marmotte provenant directement de la fosse (Ly 743/OxA-8068) $12780 \pm 75$ B.P. (Cartonnet et Naton 2000). La structure principale s'interprète comme une aire de rejets contemporaine de l'occupation. Cet aménagement circulaire se positionne à droite de l'entrée de la salle. Il couvre pour sa partie sommitale une surface de $3 \mathrm{~m}^{2}$ (carrés B4, C3 et C4), avec une profondeur atteignant $60 \mathrm{~cm}$. Cette excavation, en forme de puits, a atteint dans sa partie profonde les unités stratigraphiques inférieures contenant la faune würmienne, essentiellement des restes d'ursidés, occasionnant de ce fait une contamination (perturbation) ponctuelle. Aucune origine naturelle n'explique la présence de cette dépression, nous la considérons comme intentionnellement creusée. En effet, l'éventualité d'un creusement par des animaux fouisseurs (blaireaux, renards etc.) est une possibilité, mais le contexte topographique de la salle et la morphologie de la structure ne plaident pas en faveur de cette hypothèse. La fosse, de forme conique, assez régulière, ne présente aucune trace ou départ de terrier. Par ailleurs, aucune galerie n'a été observée dans cet ensemble stratigraphique. L'éventualité d'une bauge est également à exclure, la fosse trop profonde n'est pas assez large et surtout ne correspond pas aux aménagements en forme de cuvette constatés dans les couches inférieures. De gros blocs, difficilement manipulables par des animaux, provenant du creusement des couches sous-jacentes, ont été réutilisés pour combler la fosse après le rejet des ossements de marmottes et des artefacts endommagés et usés. De même, l'éventualité d'une fosse naturelle reste difficilement explicable sans aucun phénomène de soutirage, sans écoulement d'eau

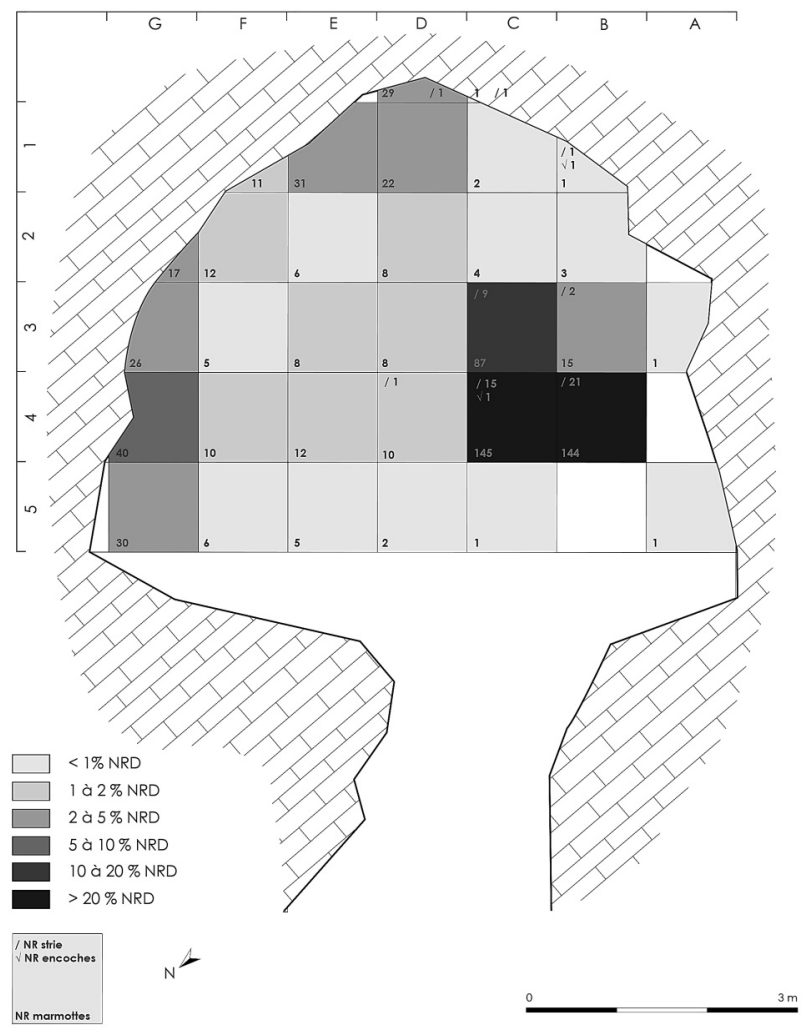

de surface ou de profondeur. Des ossements se trouvaient en fond de fosse, associés à plusieurs lames de silex ainsi qu'à des fragments de sagaies et quelques outils travaillés sur des supports en os ou bois de cervidés. Aucune connexion anatomique, même partielle, n'est constatée. On remarque l'absence de sédiment fin entre les blocs qui ont servi au comblement de la fosse. La partie supérieure de celle-ci a été colmatée avec des blocs calcaires provenant du creusement et un sédiment argileux jaune récolté dans le fond du puits. Aucun artefact produit par les occupations postérieures, mésolithiques et protohistoriques, ne s'est infiltré dans la fosse. Cette fosse compte en revanche 53,25\% des restes de marmottes de la salle 2 (fig.4). Enfin, la répartition des quelques silex recueillis sur l'intégralité de la salle n'apparaît pas comme représentative d'un quelconque atelier de débitage : très peu d'éclats corticaux, pas de nucléus et essentiellement des outils comme des grattoirs, des burins, des perçoirs ou des lamelles à dos.

\section{I LE SPECTRE FAUNIQUE DU NIVEAU MAGDALÉNIEN $2 C$}

Le niveau magdalénien $2 c$ de la Chênelaz présente un grand nombre des restes osseux appartenant à des grands mammifères (13 espèces, 1952 restes dont 1020 de marmottes), des oiseaux (47 restes, 7 espèces) (tabl. 1) et de la microfaune, insectivores et rongeurs dont l'étude a d'ores et déjà été publiée (Jeannet et Cartonnet 2000). Par ailleurs, dans cette dernière étude, il est mentionné la présence de belette (Mustela nivalis) et d'hermine (Mustela erminea) à ajouter au spectre faunique présenté ici. Il est important de souligner qu'un tiers des restes se rapportent à l'ours, dont une majorité à l'ours des cavernes (Ursus spelaeus). Les restes d'ursidés sont

\section{FIGURE 4}

La Chênelaz : répartition spatiale des restes de Marmotte (NRD = 706, NR strie = 51 , NR encoche $=2$ ).

La Chênelaz: spatial distribution of marmot remains (NRD $=706$, NR cutmarks = 51, NR notch $=2$ ). 


\begin{tabular}{|c|c|c|c|c|c|}
\hline Taxons & NRD & $\%$ NRD & $\%$ NRD (2) & NMI & NR anthr. \\
\hline Ours (Ursus spelaeus/Ursus arctos) & 699 & 35,0 & -- & -- & -- \\
\hline Loup (Canis lupus) & 5 & 0,3 & 0,4 & 1 & -- \\
\hline Renard (Vulpes vulpes) & 1 & 0,1 & 0,1 & 1 & -- \\
\hline Fouine/ Martre (Martes sp.) & 1 & 0,1 & 0,1 & 1 & -- \\
\hline Cerf (Cervus elaphus) & 58 & 2,9 & 4,5 & 5 & 1 \\
\hline Renne (Rangifer tarandus) & 46 & 2,3 & 3,5 & 3 & 10 \\
\hline Chevreuil (Capreolus capreolus) & 1 & 0,1 & 0,1 & 1 & -- \\
\hline Cervidé indéterminé & 3 & 0,2 & 0,2 & -- & -- \\
\hline Bouquetin (Capra ibex) & 48 & 2,4 & 3,7 & 5 & 11 \\
\hline Chamois (Rupicapra rupicapra) & 1 & 0,1 & 0,1 & 1 & -- \\
\hline Sanglier (Sus scrofa) & 1 & 0,1 & 0,1 & 1 & -- \\
\hline Cheval (Equus caballus) & 1 & 0,1 & 0,1 & 1 & -- \\
\hline Marmotte (Marmota marmota) & 1020 & 51,0 & 78,5 & 42 & 53 \\
\hline Léporidé (Lepus sp.) & 10 & 0,5 & 0,8 & 1 & -- \\
\hline Ongulé de petite taille & 36 & 1,8 & 2,8 & -- & -- \\
\hline Mammifère indéterminé & 21 & 1,1 & 1,6 & -- & 15 \\
\hline Aigle royal (Aquila chrysaetos) & 23 & 1,2 & 1,8 & 1 & -- \\
\hline Rapace diurne & 1 & 0,1 & 0,1 & -- & -- \\
\hline Grand tétras (Tetrao urogallus) & 2 & 0,1 & 0,2 & 1 & -- \\
\hline Tétras lyre (Tetrao tetrix) & 1 & 0,1 & 0,1 & 1 & -- \\
\hline Lagopède (Lagopus sp.) & 1 & 0,1 & 0,1 & 1 & -- \\
\hline Galliforme & 1 & 0,1 & 0,1 & 1 & -- \\
\hline Corvidé & 1 & 0,1 & 0,1 & 1 & -- \\
\hline Gros bec (Coccothraustes coccothraustes) & 2 & 0,1 & 0,2 & 1 & -- \\
\hline Oiseaux indéterminés & 15 & 0,8 & 1,2 & -- & -- \\
\hline Total & 1999 & 100 & 100 & 70 & 90 \\
\hline
\end{tabular}

\section{- TABLEAU 1 -}

Liste taxinomique des restes fauniques du niveau magdalénien (2c), hors microfaune. $\mathrm{NMI}=$ nombre minimum d'individus; $\mathrm{NRD}=$ nombre de restes déterminés; \% NRD (2) = pourcentage du nombre de restes déterminés (restes d'ursidés exclus) ; NR anthr. = nombre de restes présentant des traces d'activités anthropiques. La détermination des oiseaux a été faite par V. Laroulandie Laboratoire PACEA.

souvent de petite taille (i.e. fragments de dents définitives, dents lactéales de chute, phalanges) et proviennent des niveaux inférieurs (e.g. le niveau 6b compte à lui seul 23 individus pour 1218 restes, Schweizer 2002 ; le niveau $6 c$ en contient au moins 60). En effet, la disparition définitive de l'ours des cavernes coïncide avec le dernier refroidissement du climat au cours du Pléistocène, il y a 24000 à 20000 ans (Stuart et Lister 2007 ; Pacher et Stuart 2009 ; Baca et al. 2016). Ce cas n'est pas sans rappeler celui des grottes pyrénéennes renfermant des ossements d'ours des cavernes dans des niveaux magdaléniens. La position primaire de ces pièces est suspecte dans la mesure où ces niveaux d'occupations reposent directement sur des couches à ours ou sur des ensembles archéologiques dans lesquels l'ours est abondant (liste de sites dans Fosse et al. 2002).

Enfin des restes de léporidés présentent des traces de consommation par de petits carnivores (taille renard). Des os d'aigle appartiennent à un jeune individu probablement mort dans la cavité. Ils ne présentent
Species list of level 2c faunal remains, excluding microfauna. $N M I=$ minimum number of individuals; $N R D=$ number of determined remains; \% NRD (2) = percentage of number of determined remain (excluding the bear remains) NR anthr. = number of remains showing traces of human activities. Bird determination, V. Laroulandie, PACEA.

aucune trace de consommation à l'exception de celle de petits rongeurs. Les restes des autres espèces d'oiseaux ne portent aucun stigmate, leurs faibles effectifs ne permettant pas de caractériser leur agent d'accumulation. Ainsi si on exclut du décompte les restes d'ursidés, la marmotte représente $78,5 \%$ des restes identifiés dans le niveau magdalénien 2c.

\section{3 | LA MARMOTTE}

\section{1 | Représentation squelettique}

Le matériel de la couche $2 c$ est constitué de 1020 restes identifiables. La distribution anatomique montre que l'ensemble des éléments du squelette est présent. Le \% de survie permet d'apprécier plus précisément la représentation d'un élément anatomique en comparant son nombre minimal observé au nombre théorique attendu pour cet élément. Ce dernier est calculé d'après le nombre minimum d'individus dans la série étudiée, soit 42 dans notre cas. Par 
exemple, nous avons 13 métacarpiens II identifiés, alors que nous devrions théoriquement en avoir 84 (42 × 2), le $\%$ de survie est ici $13 \times 100 / 84=15,5 \%$ (tabl. 2 ; fig. 5 ). Le crâne, majoritairement représenté par des maxillaires et des prémaxillaires, a subi une forte fragmentation et présente un faible \% de survie. Avec 53 restes, les mandibules, par contre, font partie des éléments anatomiques bien représentés, notamment par la partie molaire du corps de la mandibule. Les os longs du squelette appendiculaire sont abondants en nombre de restes ( $44 \%$ du NRT) et leur pourcentage de survie est élevé (> 42,9\%, sauf la fibula [10,7\%]), mais on note une sous-représentation des petits os des membres (carpiens, tarsiens, métapodes et phalanges). Les éléments axiaux représentent une part négligeable dans la représentation squelettique avec un pourcentage de survie compris entre 2 et $7 \%$. Les os des ceintures sont moyennement représentés. Le nombre de scapula et de coxal est quasiment équivalent, essentiellement représentés par des parties articulaires. Les clavicules sont déficitaires. Le pourcentage de survie est donc important pour les mandibules, les os des ceintures et les os longs des membres, alors que squelette axial et les petits os des autopodes sont faiblement représentés.

Pour cette couche, le NMI de fréquence le plus élevé a été déterminé à partir des humérus et correspond à 29 individus. Le NMI de combinaison, qui tient compte de l'état d'ossification de chaque portion osseuse, correspond à 42 individus pour l'humérus, tout comme le tibia. La distribution squelettique des os de marmotte de la couche magdalénienne est assez proche de celle observée pour les sites du Vercors, sauf pour les os longs riches en viande. En effet, à la Chênelaz, le pourcentage de survie des humérus et des fémurs est non négligeable (respectivement 50 et $51 \%$ ) alors que pour le site de Colomb ce pourcentage n'est que de 7 \% (Tomé 1998). Cette sousreprésentation des humérus et des fémurs, observée à la grotte Colomb, mais aussi à la Passagère, par rapport aux autres os longs des membres impliquerait que des quartiers de viande (bras et cuisses) ont été exportés avec ces os (Tomé 1998, 2005 ; Monin et al. 2006). La distribution des portions squelettiques montre une sousreprésentation de certaines épiphyses, du squelette axial et des os des extrémités des pattes et peut s'expliquer par différents facteurs. La densité osseuse de certaines portions peut entrainer une disparition plus précoce de la matière osseuse. L'absence de ces portions peut aussi résulter du comportement lors du dépouillement et de la fracturation des os. Le rapport entre le pourcentage de survie et la densité a été effectué à partir des données de Lyman et al. (1992). Les densités utilisées sont celles fournies pour le genre Marmota. La corrélation entre le pourcentage de survie et la densité est assez bonne $(r=$ 0,2176 , ddl $=53$ ) avec un seuil de probabilité de 0,1. Cependant la sous-représentation des métapodes ne peut pas s'expliquer uniquement par une destruction d'origine post-dépositionnelle. La distribution des portions squelettiques résulterait d'une action conjointe entre la destruction progressive des portions les moins denses et le traitement spécifique de la carcasse.

\section{2 | Profil de mortalité et saison d'abattage}

\subsection{1 | Méthodes}

La caractérisation de la saison d'abattage chez la marmotte peut être établie à partir de plusieurs méthodes basées sur son cycle de croissance, sur l'évolution de la dentition et sur la croissance du cément dentaire (Munson 1984 ; Fournier 2005). À la Chênelaz, notre analyse a porté sur les stades d'éruption et d'usure dentaire et sur l'évolution de l'ossification des os chez les jeunes individus. Les méthodes d'étude de l'âge et de la saisonnalité, appliquées ici sur la couche $2 c$, s'intègrent dans un travail global, sur l'ensemble des sites des Alpes occidentales où la marmotte a été chassée au Tardiglaciaire (Gay 2015). Pour mener à bien ce travail, nous avons établi des référentiels à partir de données actuelles sur l'espèce Marmota marmota. La mise en place de ces référentiels s'est faite à partir de l'étude des collections de squelettes de marmottes comprenant 118 individus. Le premier référentiel permet d'identifier à partir de l'éruption et de l'usure dentaire l'âge des marmottes. Pour les individus de moins de deux ans et demi, ce référentiel permet également de déterminer la saison d'abattage. Le second référentiel, basé sur les mêmes individus que le premier, permet d'estimer l'âge et/ou la saison à partir du degré d'ossification des os des membres. Il faut noter que cette estimation est moins précise que le référentiel dentaire et donne un âge médian. La fiabilité de ces deux référentiels a été vérifiée par cémentochronologie et squelettochronologie. Pour plus de détails sur les méthodes et les référentiels de comparaison utilisés, ainsi que sur les nombreuses références bibliographiques, le lecteur pourra se référer au chapitre IV de la thèse d'I. Gay (2015).

\subsection{2 | Résultats}

L'observation de l'éruption et de l'usure dentaire sur les séries dentaires a permis de déterminer l'âge sur 42 mandibules et quatre maxillaires. La répartition des restes par classes d'âges est présentée dans le tableau 3. Elle ne permet pas ici de caractériser de type de chasse particulier sur une classe d'âge. Pour les mandibules, si l'ensemble des classes d'âge est bien représenté, nous observons une majorité d'adultes $(N R=24 ; N M I=15)$. Les quatre maxillaires appartiennent à deux individus adultes.

Pour la détermination de l'âge à partir des os longs nous avons utilisé le second référentiel établi à partir des stades d'ossifications des os longs et du coxal. L'âge a pu être déterminé sur 235 pièces du squelette postcrânien (humérus, radius, ulna, coxal, fémur, tibia et fibula) (Gay 2015). Pour ces éléments, le nombre de restes appartenant à des adultes est sensiblement identique à celui appartenant à des individus juvéniles (avec respectivement 111 et 124 restes). En nombre minimum d'individus, la population de la Chênelaz est dominée par les adultes (NMI $=15)$, suivi par les juvéniles âgés entre 22 mois et 3 ans $(\mathrm{NMI}=13)$ et par les juvéniles de 10 à 21 mois $(\mathrm{NMI}=10)$. Les plus jeunes, de moins de 10 mois, ne représentent que 4 individus. 


\begin{tabular}{|c|c|c|c|c|c|c|c|}
\hline & G & D & Ind. & Total & NME & NMIf/NMIC & \% Survie \\
\hline Fragment crânien & 11 & 20 & 5 & 36 & 20 & -- & -- \\
\hline Maxillaire avec dents & 2 & 2 & & 4 & 4 & 2 & 4,8 \\
\hline Incisive supérieure & 6 & 10 & & 16 & 16 & 10 & 19,0 \\
\hline P3 supérieure & & & 1 & 1 & 1 & 1 & 1,2 \\
\hline P4 supérieure & 3 & 7 & & 10 & 10 & 7 & 11,9 \\
\hline M1 supérieure & 1 & 1 & & 2 & 2 & 1 & 2,4 \\
\hline M2 supérieure & 1 & 4 & & 5 & 5 & 4 & 6,0 \\
\hline M3 supérieure & 2 & 3 & & 5 & 5 & 3 & 6,0 \\
\hline Molaire sup. indéterminée & & & 3 & 3 & 3 & -- & -- \\
\hline Mandibule sans dents & 3 & 1 & & 4 & 4 & 3 & 4,8 \\
\hline Mandibule avec dents & 25 & 23 & 1 & 49 & 48 & $25 / 36$ & 57,1 \\
\hline Incisive inférieure & 14 & 16 & & 30 & 30 & 16 & 35.7 \\
\hline D4 inférieure & 1 & & & 1 & 1 & 1 & 1,2 \\
\hline P4 inférieure & 10 & 10 & & 20 & 20 & 10 & 23,8 \\
\hline M1 inférieure & 3 & 1 & & 4 & 4 & 3 & 4,8 \\
\hline M2 inférieure & 2 & 5 & & 7 & 7 & 5 & 8,3 \\
\hline M3 inférieure & 3 & 1 & & 4 & 4 & 3 & 4,8 \\
\hline Molaire inf. indéterminée & & & 6 & 6 & -- & -- & -- \\
\hline Fragment incisive & & & 22 & 22 & -- & -- & -- \\
\hline Côte & & & 31 & 31 & 23 & 2 & 2,3 \\
\hline Clavicule & 6 & 5 & & 11 & 11 & 6 & 13,1 \\
\hline Vertèbre & & & 66 & 66 & 31 & 6 & 2,8 \\
\hline Sacrum & & & 5 & 5 & 3 & 3 & 7,1 \\
\hline Scapula & 26 & 16 & & 42 & 35 & 21 & 41,7 \\
\hline Humérus & 33 & 31 & 6 & 70 & 42 & $29 / 42$ & 50,0 \\
\hline Radius & 21 & 26 & 2 & 49 & 41 & $22 / 31$ & 48,8 \\
\hline Ulna & 24 & 22 & & 46 & 36 & $18 / 31$ & 42,9 \\
\hline Métacarpe II & 7 & 5 & 1 & 13 & 13 & 7 & 15,5 \\
\hline Métacarpe III & 10 & 7 & & 17 & 17 & 10 & 20,2 \\
\hline Métacarpe IV & 7 & 3 & & 10 & 10 & 7 & 11,9 \\
\hline Métacarpe V & 11 & 7 & & 18 & 18 & 11 & 21,4 \\
\hline Coxal & 26 & 24 & & 50 & 30 & $24 / 38$ & 35,7 \\
\hline Fémur & 24 & 18 & 16 & 58 & 43 & $17 / 30$ & 51,2 \\
\hline Tibia & 32 & 38 & 1 & 71 & 49 & $25 / 42$ & 58,3 \\
\hline Fibula & 9 & 2 & & 11 & 9 & 9 & 10,7 \\
\hline Talus & 8 & 14 & & 22 & 22 & 14 & 26,2 \\
\hline Calcanéum & 14 & 5 & & 19 & 19 & 14 & 22,6 \\
\hline Métatarse I & 3 & 4 & & 7 & 7 & 4 & 8,3 \\
\hline Métatarse II & 7 & 4 & & 11 & 10 & 7 & 11,9 \\
\hline Métatarse III & 6 & 2 & & 8 & 8 & 6 & 9,5 \\
\hline Métatarse IV & 9 & 6 & & 15 & 14 & 9 & 16,7 \\
\hline Métatarse V & 2 & 3 & & 5 & 5 & 3 & 6,0 \\
\hline Phalange I & & & 71 & 71 & 69 & 2 & 9,1 \\
\hline Phalange II & & & 9 & 9 & 9 & 1 & 1,2 \\
\hline Phalange III & 15 & 5 & 17 & 37 & 37 & 4 & 4,9 \\
\hline Métapode indéterminé & & & 19 & 19 & -- & -- & -- \\
\hline (1) & 387 & 351 & 282 & 1020 & 795 & $29 / 42$ & -- \\
\hline
\end{tabular}

- TABLEAU 2 -

Décompte et pourcentage de survie des éléments anatomiques ( $G$ = gauche, $D=$ droit, indet. $=$ indéterminé, $\mathrm{NME}=$ Nombre minimal d'éléments, $\mathrm{NMI}=$ nombre minimal d'individus de fréquence et de combinaison).
Number and percentage survival of anatomical elements $(G=$ left, $D=$ right, indet $=$ indeterminate, $M N E=$ minimum number of elements, $M N I=$ minimum number of individuals of frequency and combination). 


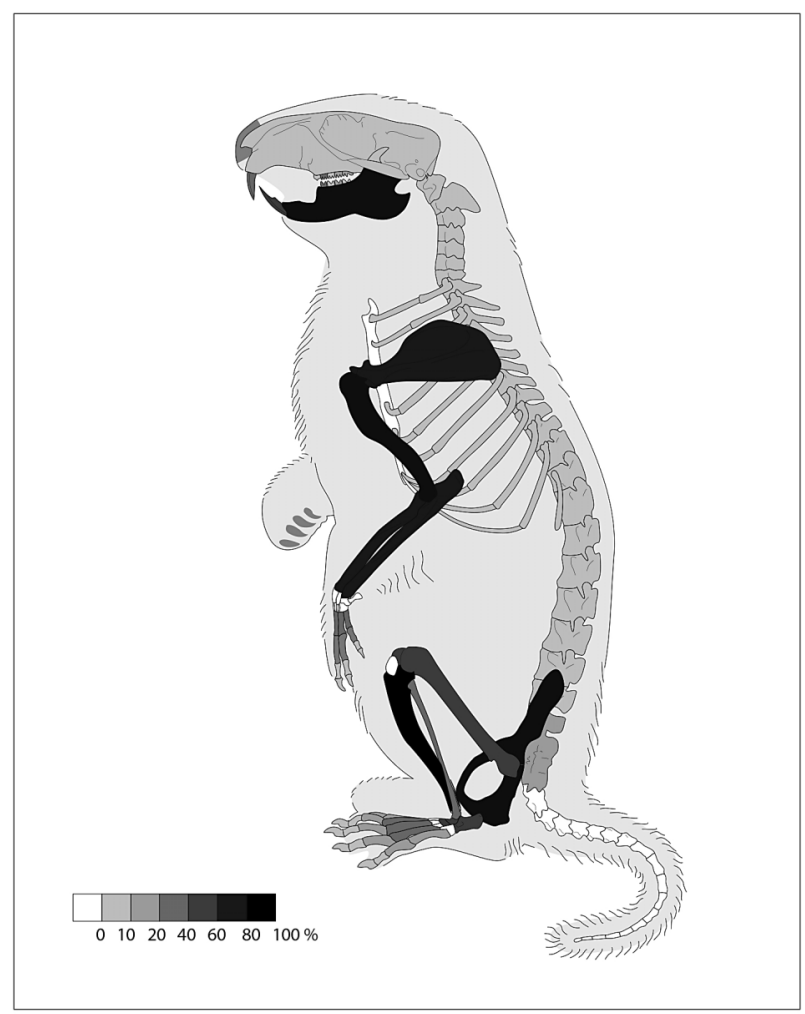

\section{FIGURE 5}

Pourcentage de survie des éléments squelettiques pour la marmotte (Définition dans le texte).

Percentage survival of skeletal elements for the marmot (Definition in the text).

\begin{tabular}{lcccc}
\hline & $\begin{array}{c}\text { Marmottons } \\
\text { (< de 10 mois) }\end{array}$ & $\begin{array}{c}\text { Juvéniles 1 } \\
\text { (De 10 à 21 mois) }\end{array}$ & $\begin{array}{c}\text { Juvéniles 2 } \\
\text { (De 22 mois à 3 ans) }\end{array}$ & $\begin{array}{c}\text { Adultes* } \\
\text { (> 3 ans) }\end{array}$ \\
\hline $\begin{array}{l}\text { Mandibule } \\
\text { Maxillaire }\end{array}$ & $7(4)$ & $9(7)$ & $2(2)$ & $24(15)$ \\
Humérus & & & & $4(2)$ \\
Radius & $2(2)$ & $12(10)$ & $11(6)$ & $22(7)$ \\
Ulna & $6(2)$ & $20(9)$ & $13(8)$ & $9(6)$ \\
Coxal & & $3(2)$ & $11(8)$ & $16(8)$ \\
Fémur & $3(2)$ & $1(1)$ & & $25(15)$ \\
Tibia & $4(3)$ & $3(3)$ & $2(2)$ & $11(8)$ \\
Fibula & $1(1)$ & $3(3)$ & $24(13)$ & $26(14)$ \\
\hline \multicolumn{1}{c}{$\%$} & $2(2)$ & $3(2)$ & $2(2)$ \\
\hline \multicolumn{1}{c}{ NMI NMI } & 4 & 10 & 13 & 15 \\
\hline
\end{tabular}

\section{- TABLEAU 3 -}

Répartition des restes de marmottes par classe d'âges, en NR et (NMI). *'espérance de vie d'une marmotte est d'une quinzaine d'années.

Le référentiel d'éruption et d'usure dentaire permet de définir la saison de mort pour les individus de moins de 29 mois. La saison d'abattage a pu être déterminée sur 18 mandibules de juvéniles. La période la mieux représentée est la fin de la bonne saison qui regroupe 13 mandibules (soit $72 \%$ des mandibules de juvéniles). Les périodes de début et de milieu de bonne saison sont représentées, avec respectivement une et quatre mandibules. Aucun reste ne caractérise la mauvaise saison.
Distribution of marmot remains by age class, in NR and (MNI). * The life expectancy of a marmot is about fifteen years.

La saison d'abattage a aussi été déterminée pour 83 restes post-crâniens. Ces restes proviennent d'individus tous morts lors de la bonne saison. Le pourcentage de restes est d'environ $20 \%$ pour le début et le milieu de bonne saison alors que la fin de bonne saison représente $59 \%$. La figure 6 représente l'estimation du nombre minimum d'individus obtenu pour chaque période à partir des mandibules et des éléments postcrâniens. Ainsi plus de la moitié des individus sont morts à la fin de la bonne saison, période correspondant à la fin de l'été et au début de l'automne alors que les marmottes ont accumulé un maximum de graisse en préparation de leur hibernation. 


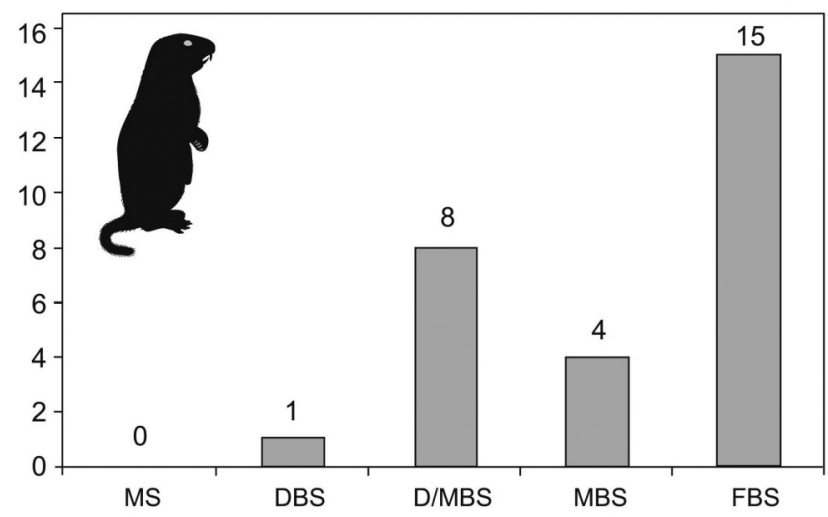

\section{3 | Les traces sur les ossements}

Dans l'ensemble, les ossements présentent un bon état de conservation. Quelques traces d'origine abiotique (weathering, agents physico-chimique et stries de charriage) se remarquent sur moins de $1 \%$ des restes. Les restes comportant des traces biotiques d'origine non-humaine représentent 4,7\% du NRD total. Quatre os portent des impacts de dents laissés par des petits carnivores, les autres traces correspondent essentiellement à des marques de dents de rongeurs. Nous avons identifié parmi ces éléments une ulna et un fémur qui portent également une fracturation d'origine anthropique. Les os rongés ont été trouvés dans un espace mal colmaté, ils sont ainsi restés accessibles aux petits carnivores et rongeurs après leur abandon.

\subsubsection{Les traces de découpe}

L'exploitation par l'homme des carcasses est attestée par la présence de stries de découpe et par la fracturation des os (dépouillement, décharnement etc.). Les stries et encoches de fracturation sont visibles sur 53 restes, soit $6,3 \%$ du nombre de restes observables (tabl. 4, fig. 4) et attestent de l'ensemble du processus de boucherie ${ }^{2}$. Hormis quatre mandibules, une scapula et un os coxal, toutes les autres pièces sont des os longs (fig.7). Ainsi, une des mandibules présente des stries sur la partie inférieure de son corps, résultant d'une opération de dépouillement de l'animal, tandis que les trois autres mandibules présentent des stries longitudinales sur la face vestibulaire entre les molaires et l'échancrure sigmoïde et correspondent à des stries de désarticulation. Les stries sur l'os coxal, situées au niveau de l'acétabulum, résultent de la désarticulation coxo-fémorale, tandis que la scapula présente des stries autour de la cavité glénoïde produites également lors de la désarticulation de l'animal. Aucune strie n'est visible sur les côtes, les vertèbres et les clavicules. Les stries visibles sur les extrémités des os longs sont courtes et liées à des opérations de désarticulation (fig. 8). Sur les diaphyses, les stries courtes transversales à l'allongement des os et les longues stries parallèles résultent du prélèvement des muscles sur ces os (opération de décharnement). Parmi les éléments striés, un peu plus de la moitié appartiennent à des

[2] La boucherie peut se définir comme une modification et une réduction (traitement) par l'homme d'une proie en parties utilisables. Dans cette définition « utilisable » signifie tous les produits d'un animal mort, non restreints à la nourriture carnée (Monchot 1996).

\section{FIGURE 6}

Répartition des marmottes en nombre minimum d'individus par saison d'abattage (MS = mauvaise saison; DBS = début de la bonne saison; $M B S=$ milieu de la bonne saison, FBS = fin de la bonne saison. La bonne saison s'étend chez la marmotte de la fin du printemps au début de l'automne).

Distribution of marmots in number of individuals per slaughter season (MS = bad season, DBS = start of the good season, MBS = mid-season, BSF = end of the good season. The good season extends to the marmot from late spring to early fall).

juvéniles dont 14 os longs du membre antérieur, un coxal et 13 os longs du membre postérieur, suggérant que les carcasses des marmottons sont exploitées comme celles des adultes. Ces incisions sont semblables à celles observées dans d'autres gisements de l'arc alpin, e.g. la grotte Colomb, l'Abri de l'Olette, l'Abri des Freydières, la grotte de la Passagère ou encore la grotte del Clusantin (Patou 1987 ; Tomé 1998, 2005 ; Monin et al. 2006 ; Romandini et al. 2012 ; Gay 2015 ; Romandini et al. 2018).

\subsection{2 | La fragmentation osseuse}

Le nombre d'éléments complets varie en fonction des éléments anatomiques. Les petits os et les dents sont généralement entiers, tout comme les mandibules, malgré une perte fréquente des zones articulaires de la branche montante. La fracturation des os longs est plus importante à proximité des extrémités articulaires libérant ainsi un nombre non négligeable de tubes diaphysaires. Cette fracturation est en partie d'origine anthropique : des fractures en spirale sur os frais, sont visibles sur 20 os longs (Villa et Mahieu 1991). Parmi les éléments anatomiques fracturés, seulement 9 pièces appartiennent à des individus de moins de 29 mois (3 humérus, 2 radius et 4 tibias).

\section{4 | DISCUSSION}

\section{1 | Exploitation des carcasses}

Les marmottes semblent avoir été introduites entières sur site où s'effectuait l'ensemble des activités de boucherie, du dépouillement au décharnement. Cette exploitation semble avoir été effectuée près de la fosse dépotoir aménagée par les Magdaléniens. La plus forte concentration en ossements se trouve en bordure de la limite C4/B4, correspondant au centre de cette fosse. Les relevés de fouilles indiquent que le comblement de la fosse fût réalisé au fur et à mesure de son utilisation suggérant des apports progressifs en carcasses de marmottes.

Néanmoins, la faible fréquence du nombre de stries observées à la Chênelaz comparée aux autres sites du Vercors (tabl.4) peut être le reflet de plusieurs facteurs: (1) une plus grande dextérité des chasseurs de la Chênelaz lors de la découpe animale laissant ainsi moins de traces sur les ossements ; (2) on ne peut exclure totalement une origine naturelle pour certains individus venus mourir dans le fond de la cavité ou provenant par intrusion des niveaux inférieurs moustériens (e.g. contamination liée au creusement de la fosse anthropique); (3) enfin des 


\begin{tabular}{lcccc}
\hline & NRO & NMI & NR stries & \% stries \\
\hline La Chênelaz, niveau 2C & 842 & 42 & 53 & 6,3 \\
Grotte Colomb & 4366 & 480 & 891 & 20,4 \\
La Passagère & 1392 & 93 & 148 & 10,6 \\
Abri l'Olette & 56 & 10 & 11 & 19,6 \\
Abri des Freydières & 559 & 41 & 123 & 22,0 \\
Abri de Bobache, couche 2 & 140 & 5 & 11 & 7,8 \\
\hline
\end{tabular}

\section{- TABLEAU 4 -}

Décompte par parties anatomiques du nombre de restes observables (NRO) de marmotte présentant des stries de boucherie (NMI = Nombre minimum d'individus)

Number of Marmot remains (NRO) showing butchery cutmarks (MNI = Minimum (informations dans Gay, 2015).

MANDIBULE

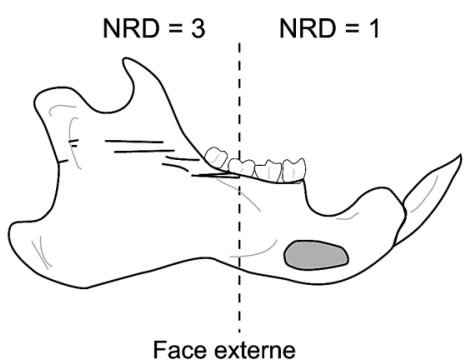

ULNA

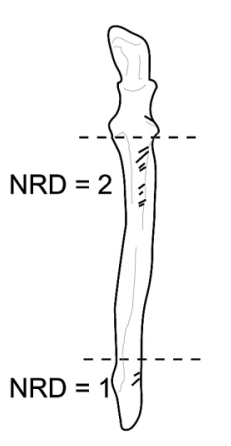

F. antérieure

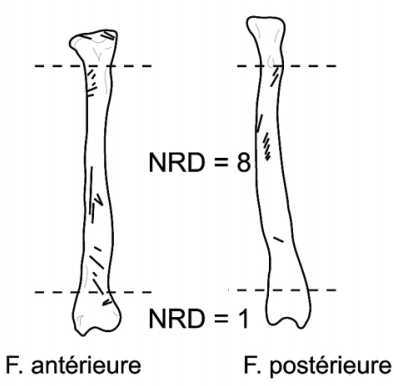

RADIUS

F. postérieure

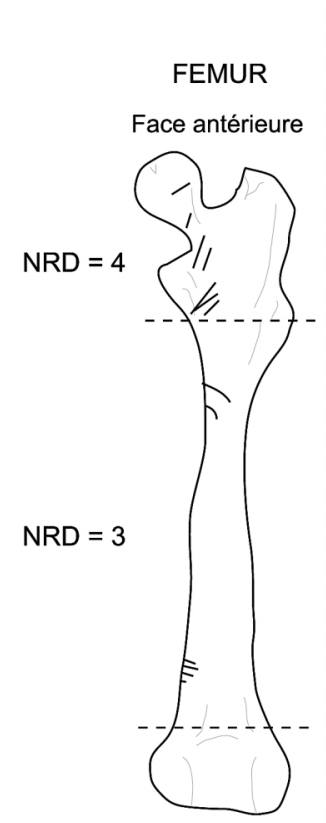

HUMERUS

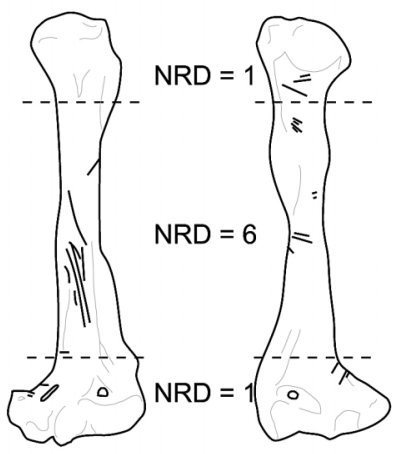

F. antérieure $\quad$ F. postérieure

COXAL

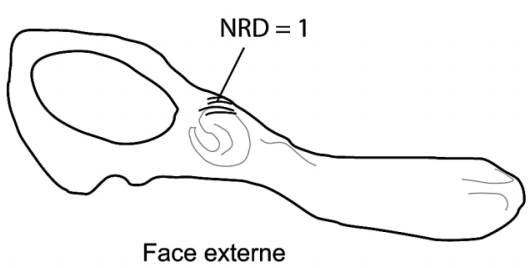

TIBIA

F. antérieure

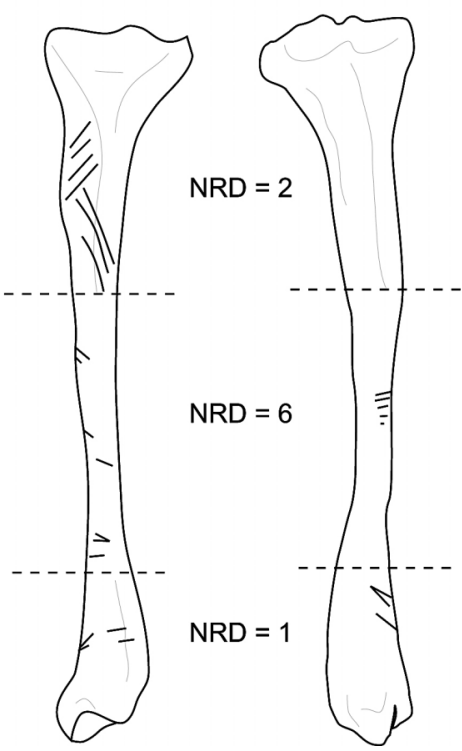

FIBULA

F. postérieure

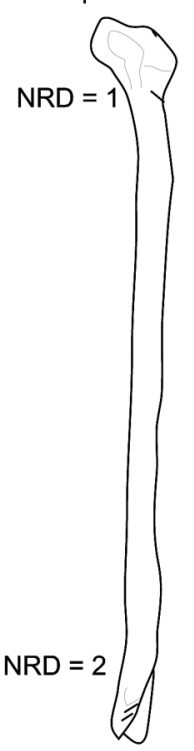

\section{FIGURE 7}

Localisation des stries de boucheries sur les mandibules, coxaux et os longs des membres antérieurs et postérieurs.

Location of butchery marks on mandibles, coxae and long bones of hindlimb and forelimb. 


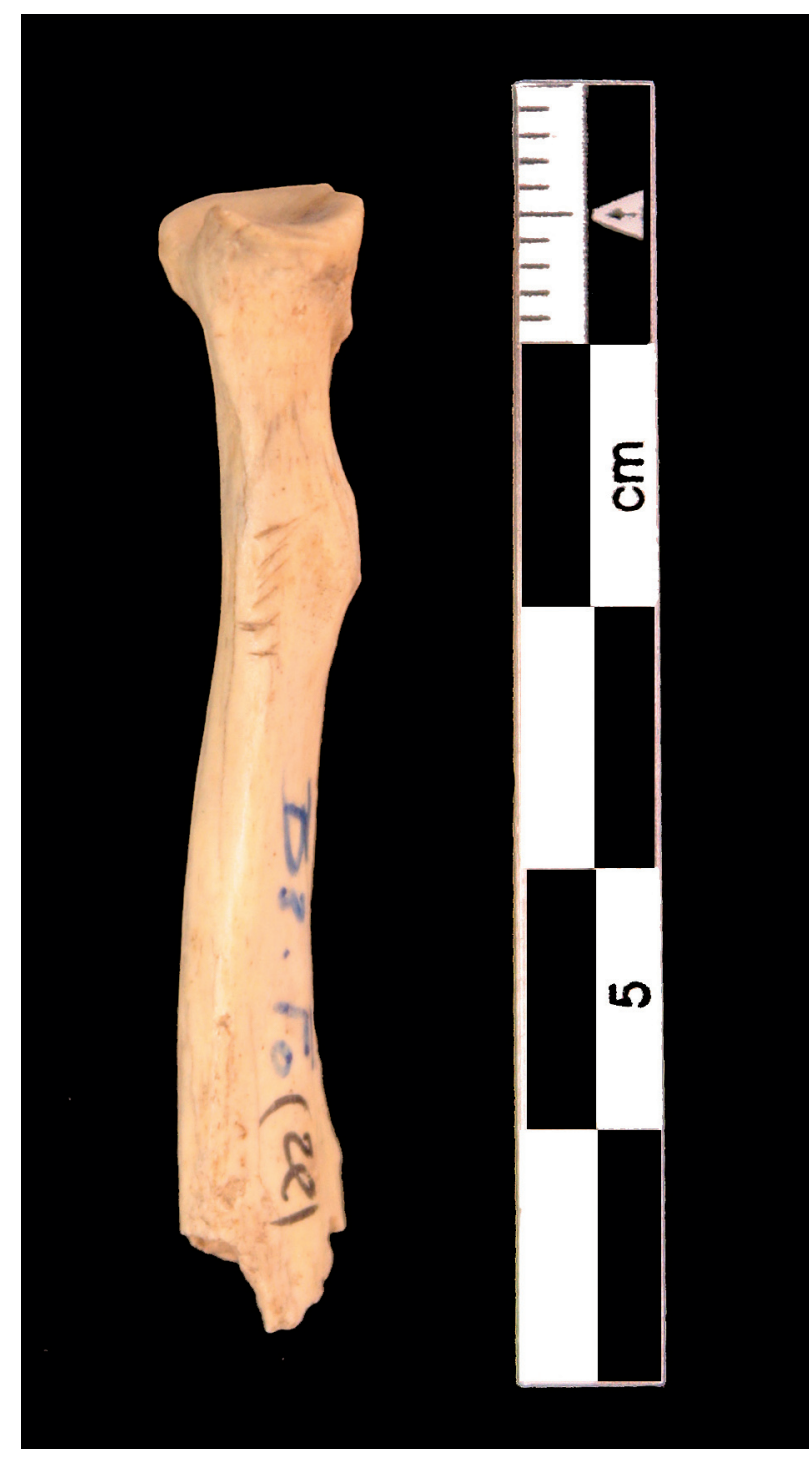

différences dans la méthode de décompte utilisée ou la taille du stock osseux à comparer peut engendrer de notables différences. Ainsi de nombreux facteurs ont été évoqués pour expliquer la variabilité des fréquences de stries au sein d'assemblages fauniques (liste complète de ces facteurs dans Chong et al. 2019).

Le nombre et la localisation des stries de désarticulation et de décharnement, associé à la fracturation intentionnelle des ossements, témoignent d'une découpe des marmottes en portions sans cuisson préalable. En effet, dans le cas contraire et quel que soit le mode de cuisson, la viande se détache facilement de l'os ne nécessitant pas un décharnement complet à l'aide d'un outil (Morel et Müller 1997). Ce désossage complet de marmottes et marmottons a certainement pour but de conserver la viande, par séchage ou par fumage, en vue d'une consommation différée (Tomé 2005 ; Monin et al. 2006).

La sous-représentation des os des extrémités (métapodes et phalanges) est à rapprocher d'un prélèvement et d'une exportation des fourrures de marmottes hors du site, comme le suggérait déjà Müller en 1914. Enfin la sousreprésentation des éléments du rachis, côtes et vertèbres, semble être liée à des phénomènes taphonomiques (e.g. conservation différentielle, piétinement). Il est néanmoins

\section{FIGURE 8}

Stries sur un radius proximal droit de marmotte.

Cutmarks on a right proximal radius of marmot.

intéressant de souligner que dans des sites à marmottes de l'île de Vancouver, en contexte cristallin et présentant de faibles sédimentations, le déficit de ces éléments a été interprété comme une exportation de ceux-ci (Nagorsen et al. 1996).

Enfin, signalons la présence de traces de sciage sur quatre incisives inférieures, en vue certainement de la préparation d'amulettes. Trois d'entre elles sont des segments distaux, la quatrième est un segment basal se trouvant encore en place dans la mandibule (Bullinger et Müller 2005).

\section{$4.2 \mid$ Chasse}

Les données ethnographiques sur la chasse à la marmotte montrent plusieurs méthodes d'acquisition de ce petit gibier. Les méthodes les plus couramment utilisées en Amérique du Nord, en Europe et en Asie, sont la capture ou le piégeage à la sortie du terrier (Drucker 1950 ; Couturier 1964 ; Perrin 1993 ; Nargorsen et al. 1996 ; Louis 1999). Les armes de jets sont très peu utilisées et présentent de nombreuses contraintes pour cette chasse. D'une part, au sein d'une colonie de marmottes, plusieurs individus sont en position de guetteurs et préviennent très rapidement leurs congénères lors de la présence d'intrus. 
Les marmottes se cachent alors dans leurs terriers. D'autre part, après une attaque, retirées à l'intérieur de leurs terriers, les marmottes ne ressortent qu'après de nombreuses heures (5h en moyenne). Ainsi, même si elle a été suggérée, notamment par Tomé et Chaix (2003) sur le matériel de la grotte Colomb, l'utilisation d'armes de jets pour ce gibier est beaucoup moins rentable que le piégeage. En termes d'acquisition, la stratégie que nous avons envisagée est le piégeage des marmottes à la sortie du terrier. L'absence de sélection des individus abattus concorde avec ce mode de capture.

Ce gibier ne devait constituer qu'un appoint, bien que sa capture soit relativement aisée du fait de la territorialité des animaux. En effet, la structure sociale de la marmotte alpine s'articule autour du groupe familial qui peut varier entre 6 à 15 individus (un groupe familial typique est composé d'un unique couple d'adultes dominants reproducteurs, d'un nombre variable d'adultes subalternes des deux sexes de deux ans et plus, et de jeunes de cohortes subséquentes, Perrin 1993 ; Perrin et al. 1993 ; Dumont 2011) et son domaine vital couvre de 2 à 5 hectares. Une chasse spécifique sur cet animal aboutirait à l'extermination presque complète du groupe le plus proche de la cavité. Il est plus probable que la marmotte constitue un gibier d'appoint au renne et au bouquetin. L'accumulation des restes de marmottes se serait alors effectué lors de multiples occupations du site, expliquant par ailleurs le dépôt progressif de ces restes dans la fosse.

\section{5 | CONCLUSION}

L'étude archéozoologique menée sur l'accumulation magdalénienne (couche $2 c$ ) des restes fauniques de la grotte de la Chênelaz confirme la domination de Marmota marmota sur les autres taxons, y compris les ongulés et carnivores identifiés lors de la fouille. La présence de marmottes dans cette couche archéologique implique que le paysage était plus ouvert qu'aujourd'hui. En effet, pour que des marmottes puissent établir une colonie plusieurs paramètres environnementaux sont nécessaires. La présence de pelouse alpine est indispensable. Elle doit être associée à un sol de type morainique ou des éboulis permettant aux marmottes de creuser leurs terriers pour l'hiver dont la profondeur est comprise entre 2 et $5 \mathrm{~m}$. La corrélation entre l'implantation de colonies de marmottes et l'abaissement de la limite supérieure de la forêt a été observée pour les sites du Tardiglaciaire du Vercors (Monin et al. 2010 ; Griggo et al. 2012 ; Gay 2015).

L'implantation du site (topographie, altitude, etc.) et les indices archéozoologiques (liste faunique, courbe d'abattage, facteurs extrinsèques sur les ossements, etc.) soulignent que l'occupation magdalénienne à la Chênelaz était orientée vers une chasse saisonnière à la marmotte, au renne et au bouquetin et plaide pour des installations de courte durée, établies à proximité des biotopes à marmottes. La grotte de la Chênelaz a été occupée au moins d'avril à octobre. Cette période semble être propice pour la chasse à la marmotte au niveau de l'arc alpin puisque l'on a retrouvé également sur plusieurs sites comme Les Freydières, L'Olette, La Passagère ou encore Colomb (Monin et al. 2010 ; Gay 2015). Toutefois, les indications saisonnières montrent que la chasse à la marmotte a été pratiquée à la Chênelaz de manière plus intensive pendant la fin de cette période, de fin août au mois d'octobre. Cette période correspond au moment le plus avantageux pour leur chasse, l'animal ayant accumulé des réserves pour l'hiver. Ces réserves sont présentes principalement sous forme de graisse dans les muscles et dans une couche de graisse sous-cutanée. C'est aussi la période pendant laquelle la fourrure est de meilleure qualité3. Des différences apparaissent dans le mode traitement de cet animal entre les sites de l'arc alpin et du Jura. Outre le reflet de l'histoire taphonomique propre à chaque site, celles-ci témoignent probablement des gestes pratiqués par les bouchers de ses différentes populations.

\section{RÉFÉRENCES BIBLIOGRAPHIQUES}

AIRVAUX J., BEAUVAL C. et PRIMAULT J. 2012 - Le site moustérien récent de La Ganne à Mazerolles et les repaires d'hyènes des Plumettes et des Rochers de Villeneuve à Lussac-les-Châteaux (Vienne). Hypothèses sur la relation Homme - Carnivores. Préhistoire du Sud-Ouest 20, p. 3-37.

BACA M., POPOVIC D., STEFANIAK K., MARCISCAZ A., URBANOWSKI M., NADACHOWSKI A. et MACKIEWICZ P. 2016 Retreat and extinction of the Late Pleistocene cave bear (Ursus spelaeus sensu lato). The science of Nature 103: 92. https:// link.springer.com/article/10.1007/s00114-016-1414-8

BEAUVAL C. 2004 - La faune des niveaux moustériens de «Chez-Pinaud »(Jonzac, Charente-Maritime, France). Première analyse. In : J. Airvaux. (dir.), Le site paléolithique de Chez-Pinaud à Jonzac, Charente-Maritime. Préhistoire du Sud-Ouest, suppl. 8, p. 125-156.

BEAUVAL C., BISMUTH T., BRUXELLES L., MALLYE J.-B. et BERTHET A.-L. 2004 - La Chapelle-aux-Saints : 1905-2004. Un siècle de recherches. Actes du XXVIe congrès préhistorique de France. Avignon, 2004, p. 21-25.

BINTZ P. et GRIGGO C. 2011 - Climats et premiers peuplements des Alpes du Nord françaises : des derniers chasseurs aux premiers paysans (15000 à 5000 ans av. JC.). Revue de primatologie. https://journals.openedition.org/ primatologie/789

BREUIL (Abbé) 1899 - Note sur un terrier de marmottes quaternaires à Couvres (Aisne). Bulletins et Mémoires de la Société d'anthropologie de Paris, tome 10, p. 521-532.

BULLINGER J. et MÜLLER W. 2005 - Nouvelles découvertes d'incisives de marmotte sciées dans des sites magdaléniens de l'arc jurassien. Industrie osseuse et parures du Solutréen au Magdalénien en Europe, Mémoire de la Société préhistorique française XXXIX, p. 347-352.

CARTONNET M. et NATON H. G. 2000 - Le magdalénien de la grotte de la Chênelaz à Hostiaz (Ain). Société préhistorique française, mémoire XXVII, p. 235-243.

[3] Outre la viande, la peau et la fourrure, la graisse et l'huile de marmottes ont été largement utilisées notamment à des fins thérapeutiques au cours des périodes historiques (nombreuses références dans Ramousse et al. 1999). 
CHALINE J. 1972 - Les rongeurs du pléistocène moyen et supérieur de France (systématique, biostratigraphie, paléoclimatologie). Éditions du Centre national de la recherche scientifique.

CHONG S., COSTAMAGNO S., SOULIER M.-C. et VAL A. 2019 Le référentiel de stries de boucherie. Palethnographie 10 (L'acquisition et le traitement des matières végétales et animales par les néandertaliens: quelles modalités et quelles stratégies ?). https://journals.openedition.org/ palethnologie/4076

COUTURIER M. 1964 - Le Gibier des montagnes françaises. France : Arthaud. 463 p.

DESBROSSE R., MARGERAND I. et PATOU-MATIS M. 1991 Quelques sites à marmottes du tardiglaciaire dans les Alpes du nord et le Jura méridional. Actes du 116e Congrès National des sociétés savantes, Chambéry, p. 365-392.

DRUCKER P. 1950 - Culture element distribution: XXVI Northwest Coast. University of California Anthropological Records 9(3), p. 157-294.

DUMONT F. 2011 - Le rôle individuel dans les interactions sociales chez la marmotte alpine (Marmota marmota). Mémoire de maîtrise de biologie, Université du Québec à Montréal (non publié)

FOSSE F., MORE P. et BRUGAL J.-P. 2002 - Taphonomie et éthologie des Ursidés pléistocènes. In: T. Tillet et L.R. Binford (eds.), L'ours et l'homme. Liège: Etudes et Recherches de l'Université de Liège 100, p. 79-101.

FOURNIER J. 2005 - Établissement d'un référentiel actualiste de saisonnalité pour la marmotte des Alpes (collection Couturier - Muséum d'Histoire Naturelle de Grenoble). Mémoire de Master 2 de recherche. Université de Provence, Ecole Doctorale : Espace, culture et société, 126 p. (non publié).

GAY I. 2015 - La saisonnalité des occupations humaines au Tardiglaciaire dans les Alpes occidentales. Thèse Doctorat, Université Aix-Marseille (non publiée).

GIACOBINI G. 1992 - Marmots and prehistoric research: a taphonomic perspective. Analisi tafonomica di resti di marmotta presenti in depositi preistorici. Aspetti metodologici e problemi interpretativi. In: B. Bassano, P. Durio, U. Gallo Orsi et E. Macchi (eds.), Proceedings of First International Symposium on Alpine Marmot (Marmota marmota) and on Genus Marmotta. Turin, p. 1-5.

GRIGGO C., GAY I. et MONIN G., 2012 - Populations de marmotte, fluctuations climatiques et activités cynégétiques au Tardiglaciaires dans le Vercors. À la rencontre des mammifères sauvages de la région Rhône-Alpes. Muséum de Grenoble. Actes des $8^{e}$ Rencontres du patrimoine naturel en Rhône-Alpes, Octobre 2010, Grenoble, France. Museum de Grenoble, p. 110-119.

GURIOLI F. BARTOLOMEI G., NANNINI N., PERESANI M. et ROMANDINI M. 2011 - Deux clavicules de marmotte épigravettiennes incisées provenant des grottes Verdi de Pradis (Alpes italiennes). Paleo 22, p. 311-318.
JEANNET M. et CARTONNET M. 2000 - La microfaune de la Chênelaz (Hostias, Ain). L'environnement et son influence sur la biométrie chez Arvicola terrestris (Rodentia, Mammalia). Revue de Paléobiologie 19(2), p. 475-492.

LAVILLE A. 1908 - La marmotte d'Eragny. Bulletins et Mémoires de la Société d'Anthropologie de Paris 9, p. 649-655.

LOUIS S. 1999 - Interactions homme/Faune sauvage. La marmotte alpine (Marmota marmota). Thèse de doctorat, Université Claude Bernard, Lyon, 126 p.

LYMAN R. L., HOUGHTON L. E., CHAMBERS A. L 1992 - The effect of structural density on marmot skeletal part representation in archaeological sites. Journal of archaeological science 19(5), p. 557-573.

MANN C.S., MACCHI E. et JANEAU G. 1993 - Alpine marmot (Marmota marmota, L.). Ibex Journal of Mountain Ecology 1, p. $17-30$

MONCHOT H. 1996 - La consommation du Mouflon (Ovis antiqua, Pommerol, 1879) au Pléistocène moyen à la Caune de l'Arago (Tautavel, Pyrénées-Orientales). Géologie méditerranéenne XXIII(2), p. 101-115.

MONIN G., GRIGGO C. et TOMÉ C. 2006 - Stratégies d'exploitation d'un écosystème alpin au Tardiglaciaire. Les chasseurs de marmottes du Vercors. In : Y. Miras et F. Surmely (eds.), Actes de la table ronde de Pierrefort « Environnement et peuplement de la moyenne montagne du Tardiglaciaire à nos jours ». Presses Universitaires de Franche-Comté, Besançon, p. 29-50.

MONIN G., MORIN A et GRIGGO G. 2007 - Hippolyte Müller (1865-1933) : pionnier oublié de l'ethnopréhistoire. Congrès du centenaire. Un siècle de construction du discours scientifique en Préhistoire. Société préhistorique française, volume 1, p. 139-155.

MONIN G., GRIGGO C. FOURNIER J. et OBERLIN C. 2010 Exploitation d'un écosystème alpin au Tardiglaciaire : les chasseurs de marmottes (Marmota marmota) du Vercors. Données environnementales, culturelles et économiques. In : S. Tzortzis et X. Delestre (eds.), Archéologie de la montagne européenne, Actes de la table ronde internationale de Gap, 29 septembre-1er octobre 2008. Bibliothèque d'Archéologie méditerranéenne et africaine, 4 : 171-185.

MOREL P. et MÜLLER W. 1997 - Hauterive-Champréveyres, 11. Un campement magdalénien au bord du lac de Neuchâtel : étude archéozoologique (secteur 1). Neuchâtel : Musée cantonal d'archéologie (Archéologie neuchâteloise 23).

MÜLLER H. 1914 - Les stations aziliennes du Vercors. Les chasseurs de marmottes. Compte Rendu de l'Association française pour l'avancement des sciences, congrès $d u$ Havre, $43^{e}$ session, p. 642-648.

MÜLLER H. 1917. Considérations sur le préhistorique de la région grenobloise. Recueil des travaux de l'institut de géographie Alpine, t. 5, p. 385-402. 
MUNSON P.J. 1984 - Teeth of juvenile woodchucks as seasonal indicators on archaeological sites. Journal of archaeological science 11(5), p. 395-403.

NAGORSEN D. W., KEDDIE G. et LUSZCZ T. 1996 - Vancouver Island marmot bones from subalpine caves: archeological and biological significance. Victoria : B.C. Park (Occasional paper $\mathrm{n}^{\circ}{ }^{4}$ ).

NATON H.-G. 1995 - La grotte de la Chênelaz (Hostias, Ain). Étude stratigraphique et sédimentologique du remplissage karstique de la période du Würm à l'actuelle. Mémoire de maîtrise de géologie, Université Joseph-Fourrier, Grenoble (non publié).

PACHER M. et STUART A.J. 2009 - Extinction chronology and palaeobiology of the cave bear (Ursus spelaeus). Boreas 38 (2), p. 189-206.

PATOU M. 1987 - Les marmottes : animaux intrusifs ou gibiers des préhistoriques du Paléolithique. Archaeozoologia 1 , p. 93-107.

PERESANI M. (dir.) 2008 - Marmotte e cacciatori del paleolitico a Pradis. Comune di Clauzetto, Università di Ferrara 130.

PERRIN C. 1993 - Organisation socio-spatiale et distribution des activités chez la Marmotte Alpine (Marmota marmota Linné 1758). Thèse de Doctorat, Université Denis Diderot, Paris.

PERRIN C, ALLAINÉ D, LE BERRE M. 1993 - Socio-spatial organization and activity distribution of the Alpine Marmot Marmota marmota: Preliminary results. Ethology 93, p. 21-30.

POHAR V. 1997 - Late glacial mammal macrofauna in Slovenia. Quärtar 47/48, p. 149-158.

RAMOUSSE R., LE BERRE M. et GIBOULET O. 1999 - La marmotte alpine. Courrier de l'environnement de l'INRA 36, p. 39-52.
ROMANDINI M., PERESANI M. GURIOLI F. et SALA B. 2012 Marmota marmota, the most common prey species at Grotta del Clusantin: insights from an unusual case-studi in the Italian Alps. Quaternary International 252, p. 184-194.

ROMANDINI M., THUN HOHENSTEIN U., FIORE I., TAGLIACOZZO A., Andrea PEREZ 1, LUBRANO V., TERLATO G. et PERESANI M. 2018 - Late Neandertals and the exploitation of small mammals in northern Italy: fortuity, necessity or hunting variability? Quaternaire 29(1), p. 61-67.

SCHWEIZER M. 2002 - Grotte de la Chênelaz (Hostias, Ain, France) : les grands mammifères de la couche 6b. Revue de Paléobiologie, Genève 21(2), p. 803-818.

STUART A.J. et LISTER A.M. 2007 - Patterns of Late Quaternary megafaunal extinctions in Europe and Northern Asia. Courier-Forschungsinstitut Senckenberg 259, p. 287-297.

TOMÉ C. 1998 - Étude de la marmotte des Alpes (Marmota marmota L.) et de son exploitation par les chasseurs du Paléolithique final de la grotte Colomb (Méaudre-Vercors France). Mémoire de maîtrise, Université Pierre Mendés France, Grenoble II (non publié).

TOMÉ C. 2005 - Les marmottes de la grotte Colomb (Vercors-France). Revue de Paléobiologie 10, p. 11-21.

TOMÉ C. et CHAIX L. 2003 - Marmot's hunting and exploitation in the western Alps and the southern Jura from late Pleistocene to Holocene. In : Ramousse R., Allaine D., Le Berre M. (éds.), Adaptative strategies and diversity in marmots / Stratégies adaptatives et diversité chez les marmottes, International Network on Marmots, p. 79-86.

TOURNEPICHE J.-F. 1996 - Les grands mammifères de Poitou-Charente. Paleo 8, p. 109-141.

VILLA P. et MAHIEU. E. 1991 - Breakage patterns of human long bones. Journal of Human Evolution 21, p. 27-48. 